\title{
Symbolic Analysis of Economical Models with Mathematica
}

\author{
A. Gálvez and A. Iglesias \\ Department of Applied Mathematics and Computational Sciences, University of \\ Cantabria, Avda. de los Castros, s/n, E-39005, Santander, Spain
}

\begin{abstract}
Functional equations is a very powerful technique to obtain consistent models in Economics. Unfortunately, there is only a few computer tools for solving functional equations. In this paper a Mathematica package, FSolve, is applied to the symbolic analysis of some economical models for price and advertising policies. The procedure is as follows: firstly, we make some assumptions about the functional structure of the functions describing the models. Such assumptions are given in terms of functional equations that account for some economical properties. Then, the package is applied to compute the solutions of these equations and check for inconsistencies. The interesting cases of the monopoly and duopoly models are discussed.
\end{abstract}

\section{Introduction}

One of the most appealing applications of the computer algebra systems (CAS) is the symbolic analysis of economical models [8, 9]. By this we mean to determine the symbolic solutions of the equations governing those models, which must be based on reasonable properties. The starting point of this process is to establish the conditions - which must be meaningful from the viewpoint of economical sciences - that those functions must satisfy necessarily. Then, the derived equations must be solved. However, it is quite hard in general to determine which are the conditions characterizing the problem and thus, some assumptions are usually taken. For instance, the functional structure of the functions describing the models is sometimes arbitrarily chosen, based either on convenience or on empirical considerations. Very often, this fact leads to absurd solutions and/or inconsistencies.

Our experience is that the functional equations is an optimal technique to overcome these undesirable situations. They provide powerful and consistent methods to describe the common sense properties of the economical functions and, simultaneously, the mathematical tools for solving the resulting equations 1, 2, 3, 4, 5. The drawback of this picture is that most of this work must be performed by hand, as there is is only a few computer tools for solving functional equations. One remarkable exception is a Mathematica package, FSolve, developed by one of the authors and described in [6. In this paper, the package will be successfully applied to the analysis of some monopoly and duopoly models for price and advertising policies. 
The structure of this paper is as follows: in Section 2 we describe briefly the package FSolve. Then, in Section 3 we analyze some economical models for price and advertising policies. The procedure is as follows: firstly, we make some assumptions about the functional structure of the functions describing the models. Such assumptions are given in terms of functional equations that account for some economical properties. Then, the package is applied to compute the solutions of these equations and check for inconsistencies. The interesting cases of the monopoly and duopoly models are discussed.

\section{The Package FSolve}

In this section the Mathematica package FSolve is briefly described. The package has been implemented a few years ago in Mathematica 3.0 [6] but updated versions for Mathematica 4.0, 4.1, 4.2 and 5.0 are also available. We start our discussion by loading the package:

In [1] : =<<FunctionalEquations 'FSolve'

which includes the command

FSolve [eqn, $\{$ functions $\},\{$ variables $\}$,options $]$

where eqn denotes the functional equation to be solved, \{functions $\}$ is the list of unknown functions, $\{$ variables $\}$ is the list of variables and options allows the users to consider different domains for the variables (see Table 1) and classes of feasible functions (see Table 2).

For instance, we can calculate the solution of the functional equation $f(x+$ $y)=g(x)+h(y)$ where $x, y \in \mathbb{R}$ and $f, g, h$ are continuous functions as:

$$
\begin{aligned}
& \text { In }[2]:=\text { FSolve }[\mathrm{f}[\mathrm{x}+\mathrm{y}]==\mathrm{g}[\mathrm{x}]+\mathrm{h}[\mathrm{y}],\{\mathrm{f}, \mathrm{g}, \mathrm{h}\},\{\mathrm{x}, \mathrm{y}\}, \text { Domain- }- \text { Real, } \\
& \text { Class->Continuous] } \\
& \begin{aligned}
\text { Out }[2]:= & \{f(x) \rightarrow C(1) x+C(2)+C(3), g(x) \rightarrow C(1) x+C(2), \\
& h(x) \rightarrow C(1) x+C(3)\}
\end{aligned}
\end{aligned}
$$

where $C(1), C(2)$ and $C(3)$ are arbitrary constants. Note that the general solution can depend on one or more arbitrary constants and even on arbitrary functions (see Out(3) and Out(4) for two examples). Note also that a single equation can determine several unknown functions (such as $f, g$ and $h$ in this example). The reader is referred to [7] for a general introduction to the theory of functional equations and their applications.

\section{$3 \quad$ Price and Advertising Policies}

In this section the package FSolve is applied to analyze some economical models for price and advertising policies. In particular, we focus on the problem of modeling the sales $S(p, v)$ of a single-product firm such that they depend on the price $p$ of its product and on the advertising expenditure $v$. Because of limitations of space, we will restrict our discussion to the cases of monopoly and duopoly models. 
Table 1. List of all valid domains used in the FSolve package

\begin{tabular}{|c|c|c|}
\hline \hline Domains & Package names & Definition \\
\hline $\mathbb{R}$ & Arbitrary & $\mathbb{R}$ \\
$\mathbb{R}$ & Real & $\mathbb{R}$ \\
$\mathbb{I}$ & Irrational & $\mathbb{R}-\mathbb{Q}$ \\
$\mathbb{Q}$ & Rational & $\mathbb{Q}$ \\
$\mathbb{Z}$ & Integer & $\mathbb{Z}$ \\
$\mathbb{R}-\{0\}$ & NonZeroReal & $\mathbb{Q}-\{0\}$ \\
$\mathbb{Q}-\{0\}$ & NonZeroRational & $\mathbb{Z}-\{0\}$ \\
$\mathbb{Z}-\{0\}$ & NonZeroInteger & $\{x \in \mathbb{R} / x \geq 0\}$ \\
$\mathbb{R}_{+}$ & RealPositiveZero & $\{x \in \mathbb{Q} / x \geq 0\}$ \\
$\mathbb{Q}_{+}$ & RationalPositiveZero & $\{x \in \mathbb{Z} / x \geq 0\}$ \\
$\mathbb{Z}_{+}$ & IntegerPositiveZero & $\{x \in \mathbb{R} / x>0\}$ \\
$\mathbb{R}_{++}$ & RealPositive & $\{x \in \mathbb{R}-\mathbb{Q} / x>0\}$ \\
$\mathbb{I}_{++}$ & IrrationalPositive & $\{x \in \mathbb{R}$, \\
$\mathbb{Q}_{++}$ & RationalPositive & $\{x \in \mathbb{Q} / x>0\}$ \\
$\mathbb{Z}_{++}$ & IntegerPositive & $\{x \in \mathbb{Z} / x>0\}$ \\
$\mathbb{R}_{-}$ & RealNegativeZero & $\{x \in \mathbb{R} / x \leq 0\}$ \\
$\mathbb{Q}_{-}$ & RationalNegativeZero & $\{x \in \mathbb{Q} / x \leq 0\}$ \\
$\mathbb{Z}_{-}$ & IntegerNegativeZero & $\{x \in \mathbb{Z} / x \leq 0\}$ \\
$\mathbb{R}_{--}$ & RealNegative & $\{x \in \mathbb{R} / x<0\}$ \\
$\mathbb{I}_{--}$ & IrrationalNegative & $\{x \in \mathbb{R}-\mathbb{Q} / x<0\}$ \\
$\mathbb{Q}_{--}$ & RationalNegative & $\{x \in \mathbb{Q} / x<0\}$ \\
$\mathbb{Z}_{--}$ & IntegerNegative & $\{x \in \mathbb{Z} / x<0\}$ \\
\hline \hline
\end{tabular}

Table 2. Classes of valid functions used in the FSolve package

\begin{tabular}{|c|c|}
\hline \hline Name & Classes of functions \\
\hline Differentiable & Differentiable \\
Invertible & Invertible \\
Increasing & Increasing \\
Decreasing & Decreasing \\
Continuous & Continuous \\
ContinuousPoint & Continuous at a point \\
Monotonous & Monotonous \\
Arbitrary & Arbitrary \\
\hline \hline
\end{tabular}

\subsection{The Monopoly Model}

Let us assume a firm such that the sales $S$ of a single product depend on the unitary price $p$ and on the advertising expenditure $v$, that is, $S=S(p, v)$. The function $S$ cannot be arbitrary, but it must satisfy the following properties:

(M1) The $S(p, v)$ function is continuous in both arguments.

(M2) $\forall v$, the $S(p, v)$ function, considered as a function of $p$ only, must be convex from below and decreasing. This implies that, for the same advertising 
expenses, any increment in the unit price of the product leads to a reduction in sales, and that its derivative decreases with $p$.

(M3) $\forall p$, the $S(p, v)$ function, considered as a function of $v$ only, must be concave from below and increasing. This implies that, for the same unit price, an increment in the advertising expenses leads to an increment in sales.

(M4) A multiplicative change in the advertising expenditure leads to an additive change in sales, that is,

$$
S(p, v w)=S(p, v)+T(p, w)
$$

where $p \geq 0, v \geq 0, w \geq 0, T(p, 1)=0$ and $T(p, w)$ is increasing with $w$.

(M5) The sales due to an increment $q$ in price are equal to the previous sales times a real number, which depends on $q$ and $v$, that is,

$$
S(p+q, v)=S(p, v) R(q, v)
$$

where $p \geq 0, p+q \geq 0, v \geq 0, R(0, v)=1$ and $R(q, v)$ is decreasing in $q$.

Eq. (1) can be solved by using the package FSolve as follows:

$$
\begin{aligned}
& \operatorname{In}[3]:= \text { em } 1=F S o l v e[S[\mathrm{p}, \mathrm{v} * \mathrm{w}]==\mathrm{S}[\mathrm{p}, \mathrm{v}]+\mathrm{T}[\mathrm{p}, \mathrm{w}],\{\mathrm{S}, \mathrm{T}\},\{\mathrm{p}, \mathrm{v}, \mathrm{w}\}, \\
&\text { Domain- }>\text { RealPositiveZero, Class->Continuous }] \\
& \text { Out }[3]:=\{S(p, v) \rightarrow \operatorname{Arb} 1(p) \log (v)+\operatorname{Arb} 2(p), T(p, w) \rightarrow \operatorname{Arb1}(p) \log (w)\}
\end{aligned}
$$

where $\operatorname{Arb1}(p)$ and $\operatorname{Arb2}(p)$ denote two arbitrary functions depending on the variable $p$. Similarly, we can solve eq. (2) as:

$$
\begin{aligned}
\operatorname{In}[4]:= & \text { em2=FSolve }[\mathrm{S}[\mathrm{p}+\mathrm{q}, \mathrm{v}]==\mathrm{S}[\mathrm{p}, \mathrm{v}] * \mathrm{R}[\mathrm{q}, \mathrm{v}],\{\mathrm{S}, \mathrm{R}\},\{\mathrm{p}, \mathrm{q}, \mathrm{v}\}, \\
& \text { Domain->RealPositiveZero, Class->Continuous }] \\
\text { Out }[4]:= & \left\{S(p, v) \rightarrow \operatorname{Arb3}(v) e^{\left.p \operatorname{Arb4(v)}, R(q, v) \rightarrow e^{q A r b 4(v)}\right\}}\right.
\end{aligned}
$$

where $\operatorname{Arb3}(v)$ and $\operatorname{Arb} 4(v)$ denote two arbitrary functions depending on the variable $v$. Once we have solved functional equations (1) and (2) separately, the general solution of the system (12)-(2) is given by:

$$
\begin{aligned}
& \operatorname{In}[5]:=\text { FSolve }[\text { Equal @@ }(\mathrm{S}[\mathrm{p}, \mathrm{v}] / \text { First }[\#] \& / @\{\mathrm{em} 1, \mathrm{em} 2\}), \\
& \{\text { Arb1, Arb2, Arb3, Arb4\}, }\{\mathrm{p}, \mathrm{v}\}] ; \\
& \operatorname{In}[6]:=\mathrm{S}[\mathrm{p}, \mathrm{v}] / . \% \\
& \text { Out }[6]:=(C(1)+C(2) \log [v]) \operatorname{Exp}[-C(3) p]
\end{aligned}
$$

where $C(1), C(2)$ and $C(3)$ are arbitrary constants. Note that in Out [6] we have no longer arbitrary functions, but arbitrary constants. This means that the parametric model is completely specified and that we can estimate its parameters $C(1), C(2)$ and $C(3)$ using empirical data.

The obtained solution shows a logarithmic increment of sales with advertising expenditures and an exponential decrease with price, in agreement with assumptions (M4) and (M5). One justification of this model of sales is the so-called Weber-Fechner law, that states that the stimuli of the intensity of perception is a linear function of the logarithm of the intensity of the stimulus. 
The previous model was proposed by Eichhorn in 1978 [5]. It can be argued, however, that the function $R$ should depend on the price $p$, instead of $v$. Thus, we can replace (M5) by:

(M6) The sales due to an increment $q$ in price are equal to the previous sales times a real number, which depends on $q$ and $p$, that is,

$$
S(p+q, v)=S(p, v) R(q, p)
$$

where $p \geq 0, p+q \geq 0, v \geq 0, R(0, p)=1$ and $R(q, p)$ is decreasing in $q$.

The general solution of (3) can be obtained by using the package FSolve as:

$$
\begin{aligned}
\operatorname{In}[7]:= & \text { em3 }=\text { FSolve }[\mathrm{S}[\mathrm{p}+\mathrm{q}, \mathrm{v}]==\mathrm{S}[\mathrm{p}, \mathrm{v}] * \mathrm{R}[\mathrm{q}, \mathrm{p}],\{\mathrm{S}, \mathrm{R}\},\{\mathrm{p}, \mathrm{q}, \mathrm{v}\}, \\
& \text { Domain- }>\text { RealPositiveZero,Class }->\text { Continuous }] \\
\text { Out }[7]:= & \left\{S(p, v) \rightarrow \operatorname{Arb5}(p) \operatorname{Arb6}(v), R(q, p) \rightarrow \frac{\operatorname{Arb5}(p+q)}{\operatorname{Arb5}(p)}\right\}
\end{aligned}
$$

Alternatively, we can assume a multiplicative, instead of an additive, change in the price $p$ and we can question whether or not choosing between one of these assumptions influences the resulting model. In other words, we can assume:

(M7) The sales due to a multiplicative change ( $w$ times) in the price are equal to the previous sales times a real number, which depends on $w$ and $p$, that is,

$$
S(p w, v)=S(p, v) R(w, p)
$$

where $p \geq 0, w \geq 0, v \geq 0, R(1, p)=1$ and $R(w, p)$ is decreasing in $w$.

$$
\begin{aligned}
\operatorname{In}[8]:= & \text { FSolve }[\mathrm{S}[\mathrm{p} * \mathrm{w}, \mathrm{v}]==\mathrm{S}[\mathrm{p}, \mathrm{v}] * \mathrm{R}[\mathrm{w}, \mathrm{p}],\{\mathrm{S}, \mathrm{R}\},\{\mathrm{p}, \mathrm{v}, \mathrm{w}\}, \\
& \text { Domain->RealPositiveZero,Class->Continuous] } \\
\text { Out }[8]:= & \left\{S(p, v) \rightarrow \operatorname{Arb5}(p) \operatorname{Arb} 6(v), R(w, p) \rightarrow \frac{\operatorname{Arb5}(p w)}{\operatorname{Arb5}(p)}\right\}
\end{aligned}
$$

Note that the $S$ functions in Out[7] and Out[8] are identical. Thus, equations (3) and (41) are equivalent. Consequently, the above mentioned two assumptions (M6) and (M7) lead to the same model.

Now, the solution of the system (1)-(3) can be obtained as:

In $[9]:=$ FSolve $[$ Equal @@ $(\mathrm{S}[\mathrm{p}, \mathrm{v}] /$. First $[\#] \& / @\{\mathrm{em} 1, \mathrm{em} 3\})$,

$$
\{\operatorname{Arb} 1, \operatorname{Arb} 2, \operatorname{Arb} 5, \operatorname{Arb} 6\},\{\mathrm{p}, \mathrm{v}\}]
$$

Out $[9]:=\begin{aligned} &\left\{\operatorname{Arb2}(p) \rightarrow \frac{C(4)}{C(3)} \operatorname{Arb1}(p), \operatorname{Arb5}(p) \rightarrow \frac{-\operatorname{Arb1}(p)}{C(3)},\right. \\ &\operatorname{Arb6}(p) \rightarrow-C(3) \log (p)-C(4)\}\end{aligned}$

which leads to the model:

$$
\begin{aligned}
& \operatorname{In}[10]:=\mathrm{S}[\mathrm{p}, \mathrm{v}] / . \% \\
& \operatorname{Out}[10]:=\operatorname{Arb1}(p)\left[\log (v)+\frac{C(4)}{C(3)}\right]
\end{aligned}
$$

where the function $\operatorname{Arb1}(p)$ and the constants $C(3)$ and $C(4)$ are arbitrary. For this solution to satisfy assumptions (M2) and (M3) above, $\operatorname{Arb1}(p)$ must be 
convex from below and decreasing. Note that $\log (v)+\frac{C(4)}{C(3)}$ is increasing. We also remark that model in Out[10] is more general than model in Out[6]. In fact, the resulting model is not completely specified because it depends on arbitrary functions. This means that new requirements might be established.

\subsection{The Duopoly Model}

Assume now that we have two different firms that compete in the market. Assume also that the sales $S$ of the product by firm 1 depend on the unit prices $p$ and $q$ and on the advertising expenditures $u$ and $v$, of the two firms, that is, $S=S(p, q, u, v)$. The function $S(p, q, u, v)$ must satisfy the following properties:

(D1) The $S(p, q, u, v)$ function is continuous in all arguments.

(D2) $S(p, q, u, v)$ is increasing in $q$ and $u$.

(D3) $S(p, q, u, v)$ is decreasing in $p$ and $v$.

(D4) A multiplicative change in the advertising expenditure of firm 1 leads to an additive change in sales, that is,

$$
S(p, q, u w, v)=S(p, q, u, v)+T(p, q, w, v)
$$

(D5) The sales due to an increment $r$ in price of firm 1 are equal to the previous sales times a real number, which depends on $r$ and $p$, that is,

$$
S(p+r, q, u, v)=S(p, q, u, v) R(r, p, q, v)
$$

where $p \geq 0, p+r \geq 0, v \geq 0$ and $R(0, p, q, v)=1$.

The general solution of the system (5)-(6) is given by the following sequence of calculations: firstly, we compute the functions $S, T$ and $R$ of the previous equations, and then we apply the outputs to calculate the functional structure of function $S$.

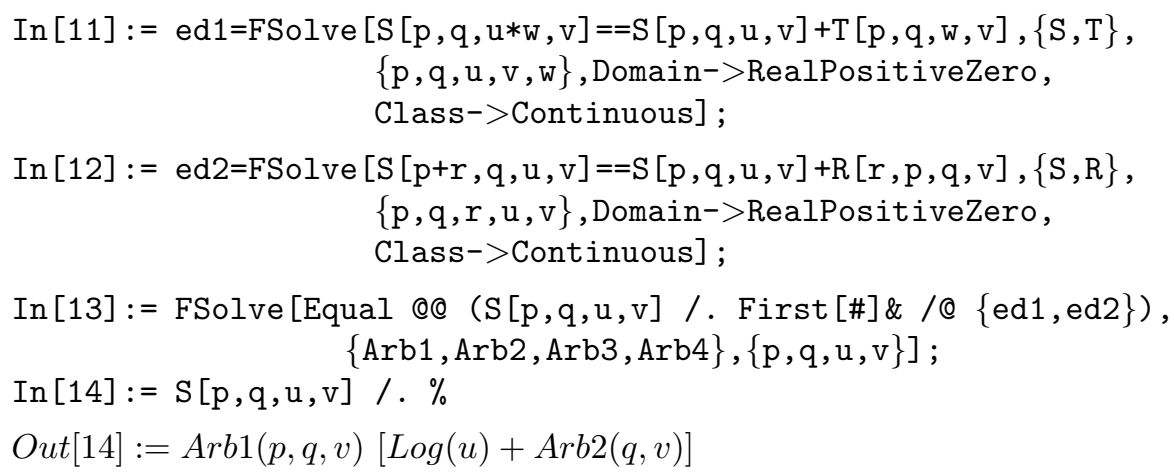

where $\operatorname{Arb1}(p, q, v)$ and $\operatorname{Arb2}(q, v)$ are arbitrary functions. In addition we can consider the following assumption:

(D6) The total sales of both firms is a constant $K$, that is,

$$
S(p, q, u, v)+S(q, p, v, u)=K
$$


which, using the previous output, leads to

$$
\begin{aligned}
\operatorname{In}[15]:= & \text { FSolve }[((\mathrm{S}[\mathrm{p}, \mathrm{q}, \mathrm{u}, \mathrm{v}]+\mathrm{S}[\mathrm{q}, \mathrm{p}, \mathrm{v}, \mathrm{u}]) / . \%)==\mathrm{K},\{\text { Arb1, Arb2 }\}, \\
& \{\mathrm{p}, \mathrm{q}, \mathrm{u}, \mathrm{v}\}, \text { Domain- }>\text { RealPositiveZero, Class->Continuous }] \\
& / / \text { FSimplify; } \\
\operatorname{In}[16]:= & \mathrm{S}[\mathrm{p}, \mathrm{q}, \mathrm{u}, \mathrm{v}] / . \%
\end{aligned}
$$$$
\text { Out }[16]:=\frac{1}{\operatorname{Arb7}(p)+\operatorname{Arb7}(q)}\left[\log \left(\frac{u}{v}\right)+K \operatorname{Arb7}(q)\right]
$$

where $\operatorname{Arb7}(p)$ is an arbitrary but increasing function of $p$. The physical interpretation of this model is as follows: if the advertisement expenditures of both firms are coincident, the sales are proportional to the ratios $\frac{A r b 7(q)}{\operatorname{Arb7}(p)+\operatorname{Arb7}(q)}$ and $\frac{A r b 7(p)}{A r b 7(p)+A r b 7(q)}$ for firms 1 and 2, respectively. On the other hand, the advertisement expenditures influence sales directly proportional to the logarithm of the ratio $\frac{u}{v}$ and inversely proportional to $\operatorname{Arb7}(p)+\operatorname{Arb7}(q)$.

We can now consider two additional assumptions:

(D7) The sales $S(p+r, q+s, u, v)$ of firm 1 due to increments $r$ and $s$ in the prices of firms 1 and 2 , respectively, are the initial sales $S(p, q, u, v)$ of firm 1 times two factors which consider the associated reduction and increments due to these two changes, that is,

$$
S(p+r, q+s, u, v)=S(p, q, u, v) U(r, p, q) V(s, p, q)
$$

(D8) The sales $S(p, q, u+r, v+s)$ of firm 1 due to increments $r$ and $s$ in the advertisement expenditures of firms 1 and 2, respectively, are the initial sales $S(p, q, u, v)$ of firm 1 times two factors which consider the associated increments and decrements due to these two changes, that is,

$$
S(p, q, u+r, v+s)=S(p, q, u, v) U(r, u, v) V(s, u, v)
$$

Combining now (D7) and (D8) and solving the system of equations (8)-(9), we get:

$$
\begin{aligned}
& \operatorname{In}[17]:=\text { FSolve }[S[p+r, q+s, u, v]==S[p, q, u, v] * U[r, p, q] * V[s, p, q] \text {, } \\
& \{\mathrm{S}, \mathrm{U}, \mathrm{V}\},\{\mathrm{p}, \mathrm{q}, \mathrm{r}, \mathrm{s}, \mathrm{u}, \mathrm{v}\}, \text { Domain->RealPositiveZero, } \\
& \text { Class->Continuous] ; } \\
& \operatorname{In}[18]:=\text { FSolve }[\mathrm{S}[\mathrm{p}, \mathrm{q}, \mathrm{u}+\mathrm{r}, \mathrm{v}+\mathrm{s}]==\mathrm{S}[\mathrm{p}, \mathrm{q}, \mathrm{u}, \mathrm{v}] * \mathrm{U}[\mathrm{r}, \mathrm{u}, \mathrm{v}] * \mathrm{~V}[\mathrm{~s}, \mathrm{u}, \mathrm{v}] \text {, } \\
& \{\mathrm{S}, \mathrm{U}, \mathrm{V}\},\{\mathrm{p}, \mathrm{q}, \mathrm{r}, \mathrm{s}, \mathrm{u}, \mathrm{v}\}, \text { Domain->RealPositiveZero, } \\
& \text { Class->Continuous] ; }
\end{aligned}
$$$$
\text { In }[19]:=\text { FSolve }[\text { Equal @@ }(\mathrm{S}[\mathrm{p}, \mathrm{q}, \mathrm{u}, \mathrm{v}] / \text {. First }[\#] \& / @\{\% \%, \%\}) \text {, }
$$$$
\{\text { Arb1 , Arb2 , Arb3, Arb6, Arb7, Arb8 }\},\{p, q, u, v\}] \text {; }
$$

$\operatorname{In}[20]:=\mathrm{S}[\mathrm{p}, \mathrm{q}, \mathrm{u}, \mathrm{v}] / . \%$

Out $[20]:=\operatorname{Arb1}(p) \operatorname{Arb2}(q) \operatorname{Arb6}(u) \operatorname{Arb7}(v)$ 
where the functions $\operatorname{Arb1}(p)$ and $\operatorname{Arb7}(v)$ are decreasing and the functions $\operatorname{Arb2}(q)$ and $\operatorname{Arb6}(u)$ are increasing, but otherwise arbitrary. The physical interpretation of this model is that all the factors (prices and advertisement expenditures) act independently and contribute to the total sales of firm 1 as a factor which is less than 1 and decreasing for $p$ and $v$ and greater than 1 and increasing for $q$ and $u$.

\section{References}

1. Aczél, J.: On a system of functional equations determining price and productivity indices. Utilitas Math. 7 (1975) 345-362

2. Aczél, J.: Measurement in economics: theory and applications of economic indices. Physica, Heidelberg (1988) 3-17

3. Aczél, J., Eichhorn, W.: Systems of functional equations determining price and productivity indices. Utilitas Math. 5 (1974) 213-226

4. Boulding, W., Lee, E., Staelin, R.: Mastering the mix: do advertising, promotion and sales force activities lead to differentiation?. Journal of Marketing Research 31 (1994) 159-172

5. Eichhorn, W.: Functional Equations in Economics. Addison Wesley, Redwood City, CA (1978)

6. Castillo, E., Iglesias, A.: A package for symbolic solution of real functional equations of real variables. Aequationes Mathematicae, 54 (1997) 181-198

7. Castillo, E., Iglesias, A., Ruiz-Cobo, R.: Functional Equations in Applied Sciences. Elsevier Pub., Amsterdam (2005)

8. Montoro, J.D., Paz, J.V.: Detecting macroeconomic chaos. In: Keranen, V., Mitic, P., Hietamaki, A. (eds): Innovations in Mathematics. Proceedings of the Second International Mathematica Symposium, IMS'97. Computational Mechanics Publications, Southampton (1997) 353-360

9. Paz, J.V., Montoro, J.D.: Classic economic dynamics: a neo-ricardian approach to economic growth and income distribution by means of Mathematica. In: Keranen, V., Mitic, P. (eds): Mathematics with Vision. Proceedings of the First International Mathematica Symposium, IMS'95. Computational Mechanics Publications, Southampton (1995) 299-308 